\title{
Morfologia do plexo lombossacral da jaguatirica (Leopardus pardalis)
}

\author{
Jessica Albuquerque Lopes ${ }^{1}$ \\ Lara Cochete Moura Fé ${ }^{1}$ \\ Ana Rita de Lima ${ }^{1}$ \\ Luiza Corrêa Pereira ${ }^{2}$ \\ Érika Branco ${ }^{1 *}$ \\ ${ }^{1}$ Universidade Federal Rural da Amazônia \\ Avenida Presidente Tancredo Neves, 2501, CEP 66077-530, Belém - PA, Brasil \\ ${ }^{2}$ Área de Mina Bauxita, Vale Paragominas - PA, Brasil \\ * Autor para correspondência \\ ebranco.ufra@gmail.com
}

Submetido em 21/05/2012

Aceito para publicação em 03/09/2012

\section{Resumo}

Conhecido popularmente como jaguatirica, o Leopardus pardalis está presente no Brasil, em todos os ecossistemas, mas preferencialmente nas matas ciliares e florestas. Este trabalho objetivou elucidar aspectos macroscópicos referentes à anatomia do plexo lombossacral. Foram utilizados três animais, sendo dois machos e uma fêmea, provenientes da área de Mina Bauxita - Paragominas-PA, doados após morte por atropelamento ao Laboratório de Pesquisa Morfológica Animal (LaPMA), da Universidade Federal Rural da Amazônia. Os animais foram fixados em solução aquosa de formaldeído a $10 \%$, seguido de dissecação dos membros pélvicos com o objetivo de expor os nervos, removendo-se parcialmente alguns músculos, dissecando-se a origem do plexo desde a intumescência lombar. Em dois animais, o nervo femoral apresentou origem no quarto nervo lombar (L4), seguindo e se transformando em nervo safeno. O nervo obturatório e o nervo isquiático tiveram origem no último nervo lombar (L5), porém, este último se dividiu em ramos originando os nervos tibial e fibular comum, originando dorsalmente os nervos glúteo caudal e glúteo cranial.

Palavras-chave: Anatomia; Leopardus pardalis; Plexo lombossacral; Sistema nervoso

\section{Abstract}

Morphology of the lumbosacral plexus of the ocelot (Leopardus pardalis). Popularly known as the ocelot, Leopardus pardalis occurs throughout Brazil in all ecosystems, but prefers riparian regions and forests. The objective of this study was to learn more about the macroscopic, anatomical aspects of the plexus lumbossacral of this species. Three specimens were studied, two males and one female, from the region near the Bauxite Mine in Paragominas, PA. The specimens were donated to the Laboratório de Pesquisa Morfológica Animal (LaPMA) at UFRA after being run over (authorization numbers 485/2009 and 522/2009). The animals were fixed in an aqueous solution of $10 \%$ formaldehyde and then the hind limb was dissected by removing some muscles to expose the nerves. In two animals, the femoral nerve originated in the fourth lumbar nerve (L4) 
and transformed into the saphenous nerve. The obturator nerve and sciatic nerve originated in the last lumbar nerve (L5), and the latter was divided into branches that formed the tibial and common peroneal nerves, which dorsally formed the cranial gluteal and caudal gluteal nerves.

Keywords: Leopardus pardalis; Lombussacral plexus; Morphology; Nerves

\section{Introdução}

O Leopardus pardalis (Linnaeus, 1758), conhecido como Jaguatirica, é uma espécie de porte médio com de $77,3 \mathrm{~cm}$ de comprimento, cauda proporcionalmente curta (cerca de $46 \%$ do comprimento da cabeça e do corpo), com média de $35,4 \mathrm{~cm}$ e peso em torno de $11 \mathrm{~kg}$. Apresenta corpo esbelto com a cabeça e patas grandes (OLIVEIRA; CASSARO, 1999).

A sua pelagem é curta e marcada com rosetas que tendem a se unir na lateral do corpo, formando listras horizontais e correndo em cadeias paralelas, o que em alguns indivíduos pode ser pouco distinguível. $\mathrm{O}$ pêlo na região da nuca cresce ao contrário daquele do resto do corpo, em direção cranial e não caudal. A cor de fundo da pelagem é muito variável, indo do cinza ao amarelo e castanho-ocre, as marcas de um lado do animal são diferentes das do outro lado e possibilitam a individualização de animais dessa espécie. $O$ ventre é claro com pintas negras, e a cauda curta, com anéis pretos. Como a maioria dos gatos selvagens brasileiros, possui uma mancha clara e redonda na face caudal das orelhas (CUBAS, 2007).

A sua dieta baseia-se principalmente em pequenos animais, como roedores e aves. A área de vida é semelhante àquela de outros felinos solitários, sendo que o território dos machos se sobrepõe ao de várias fêmeas. Esta espécie faz parte da lista oficial dos animais ameaçados de extinção do Instituto Brasileiro do Meio Ambiente e dos Recursos Naturais Renováveis (IBAMA), em função principalmente da destruição de seu hábitat (MACHADO et al., 1998).

A jaguatirica distribui-se por todos os países da América Central e do Sul até o norte da Argentina, com exceção do Chile, além do sudoeste do Texas (EUA) até porções do México. No Brasil, está presente em todas as regiões, exceto o Sul do Rio Grande do Sul. Com relação à escolha de habitat, possui uma grande plasticidade, ocupando cerrados, caatingas, pantanal, pampas, florestas tropicais, subtropicais e matas ciliares, habitando ainda matas primárias e secundárias, possivelmente tendo preferência por áreas de vegetação (SILVA, 1984; REDFORD; EISENBERG, 1992; OLIVEIRA, 1994; NOWELL; JACKSON, 1996; OLIVEIRA; CASSARO, 2005).

Quanto ao plexo lombossacral, objeto deste estudo, este é quem dá origem aos nervos do membro pélvico, sendo um prolongamento do plexo contínuo. Está situado caudalmente ao músculo psoas maior e cranialmente aos processos transversos das vértebras lombares e sacral, no qual sua localização faz face ao músculo piriforme sobre a parede posterior da pelve. A união destes dois plexos representa a origem dos nervos que se destinam aos membros pélvicos. Geralmente, começa com o ramo ventral do quarto nervo lombar e termina com o do segundo sacral (L4-S2), portanto, tem uma raiz adicional em espécies que possuem sete nervos lombares. O plexo sacral tem origem nos segmentos da medula espinhal, que se distribuem para os membros pélvicos e para as vísceras da região (DYCE, 2004).

Raros são os estudos neuroanatômicos em felinos selvagens, tornando a literatura que envolve especialmente a formação de plexos nervos algo inexistente, desta forma, objetivou-se descrever a morfologia do plexo lombossacral da jaguatirica, alavancando os conhecimentos médicos veterinários, que serão de suma importância tanto em procedimentos de técnica de anestesia peridural lombar, quanto em situações que envolvam as cirurgias ortopédicas de membros pélvicos desta espécie, bem como contribuindo para o estudo da anatomia comparada.

\section{Material e Métodos}

Foram utilizados três Leopardus pardalis (uma fêmea e dois machos) provenientes da área de Mina 
Bauxita, na cidade de Paragominas, doados ao Laboratório de Pesquisa Morfológica Animal (LaPMA), da Universidade Federal Rural da Amazônia (UFRA) após morte por atropelamento. Os animais foram fixados em solução aquosa de formoldeído a $10 \%$ tanto por via intramuscular quanto por via intracavitária e conservado nesta mesma solução por um período mínimo de sete dias.

A preparação da peça anatômica foi realizada por dissecação do segmento ocupado pelo plexo lombossacral, junto aos forames intervertebrais em ambos os antímeros, preservando-se seus componentes. Buscou-se expor o máximo possível cada vértebra lombar e sacral, a fim de melhor visualizar os ramos ventrais da medula espinhal que originam o plexo lombossacral. Desta forma, partes das vísceras abdominais e pélvicas foram removidas, assim como o tecido adiposo. Os músculos psoas maior e psoas menor foram parcialmente seccionados para facilitar a dissecação.

A nomenclatura adotada foi baseada na Nomenclatura Anatômica Veterinária (INTERNATIONAL COMMITTEE ON VETERINARY GROSS ANATOMICAL NOMENCLATURE, 2005).

\section{Resultados}

O plexo lombossacral da $L$. pardalis constituiuse dos nervos femoral, safeno, obturatório, isquiático, tibial, fibular comum, glúteo cranial e caudal. Assim, o plexo lombossacral da jaguatirica se originou das comunicações estabelecidas entre os ramos ventrais dos dois últimos nervos lombares (L4 e L5) e dos dois primeiros sacrais (S1 e S2) (Figura 1).

Ao nível de L4, observou-se que o nervo femoral, desde sua origem, acompanhou o trajeto do músculo de psoas maior, e em seguida nivelou-se a altura da tíbia, originando o nervo safeno, o qual se aprofundou alcançando no músculo quadríceps da coxa. Em sequência, ao nível de L5, originou-se o nervo obturatório, que seguiu seu trajeto até encontrar o músculo reto femoral (Figura 2).

O nervo isquiático localizou-se distalmente ao plexo lombossacral tendo origem no último nervo lombar (L5) e no primeiro sacral (S1). Passou entre os músculos glúteos médio e profundo e seguiu lateralmente se dividindo em dois ramos terminais, os nervos tibial e fibular comum. O nervo tibial emitiu ramos que inervaram os músculos pélvicos. $\mathrm{O}$ nervo

FIGURA 1: Fotomacrografia do plexo lombossacral do Leopardus pardalis (vista dorsal), em que se observa o quarto e quinto nervos lombares e primeiro e segundo nervos sacrais, sendo projetados de L4, L5, S1 e S2.

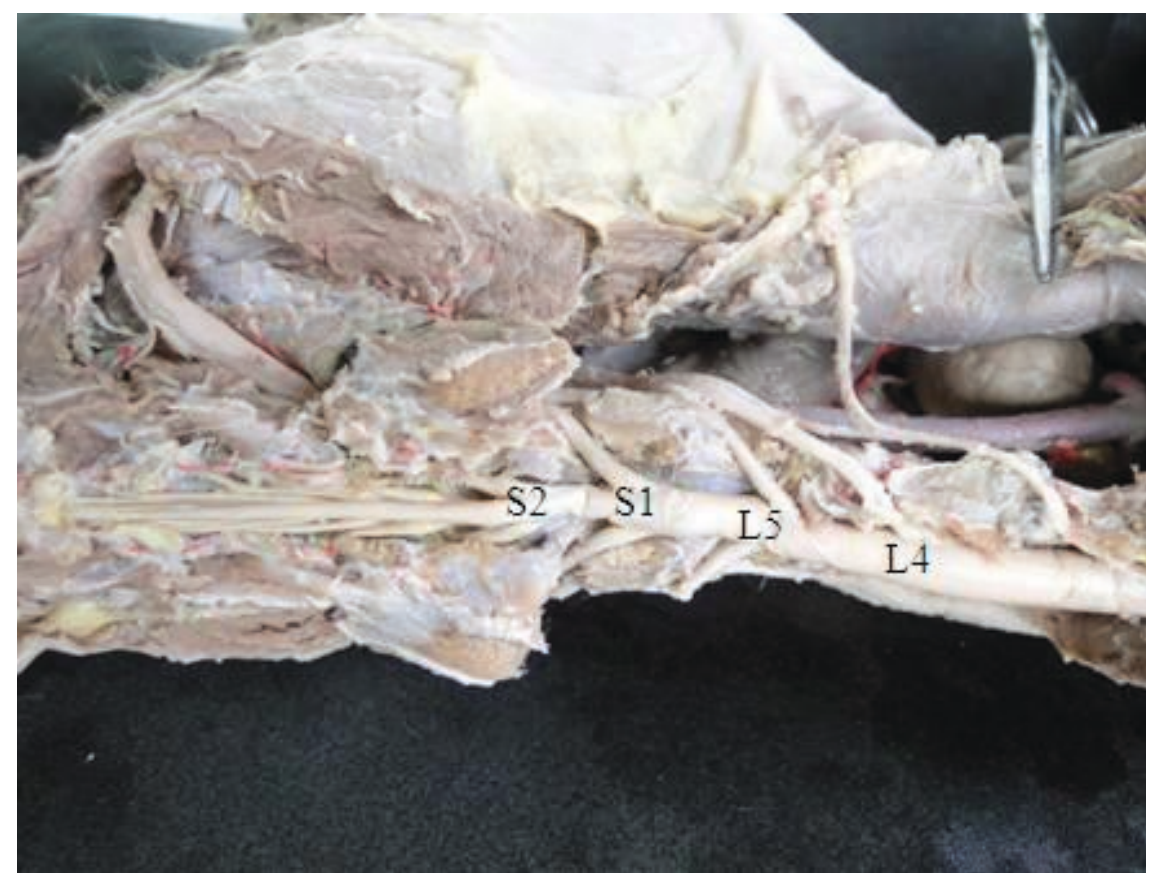


fibular comum separou-se e cruzou por cima da cabeça lateral do músculo gastrocnêmio infiltrando-se assim na musculatura (Figura 3 e 4).

Os nervos glúteo cranial e glúteo caudal originaram do nervo isquiático e emitiram ramos para inervar os músculos glúteos médio e profundo (Figura 5).

FIGURA 2: Fotomacrografia da vista medial do plexo lombossacral do Leopardus pardalis, no qual se observa o nervo femoral (NF) partindo de L4 e originando o nervo safeno (NS) que se aprofunda no músculo quadríceps (MQ), e o nervo obturatório (NO) partindo da sua origem L5.

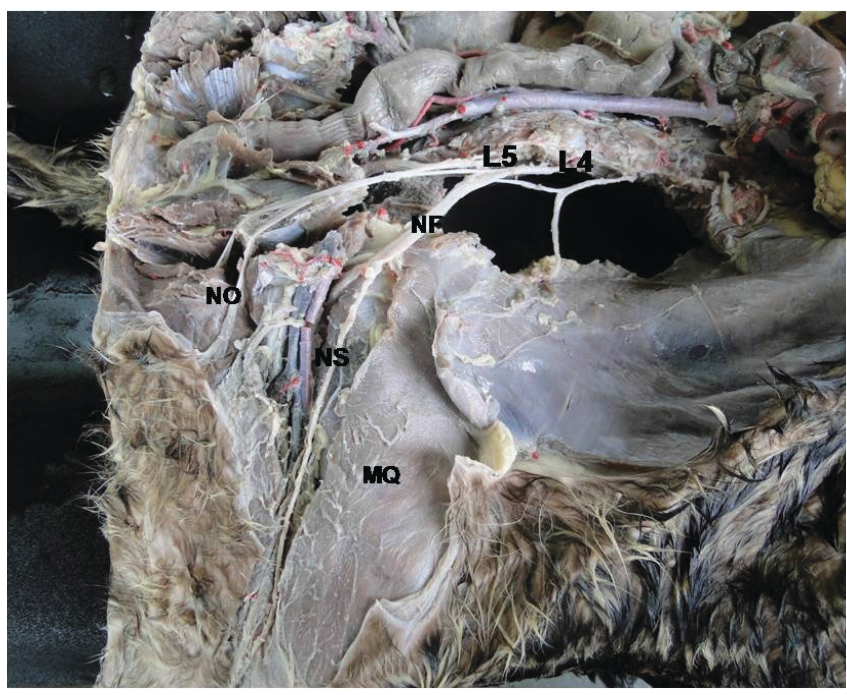

FIGURA 3: Fotomacrografia do plexo lombossacral do Leopardus pardalis (vista dorsal), em que se observa a origem do nervo isquiático $(\mathrm{NI})$, seguindo trajeto pelo músculo glúteo médio (MGm) parcialmente seccionado, o qual surge no nervo lombar L5, situado na quinta vértebra lombar (VL5) e primeiro sacral (S1), situado na primeira vértebra sacral (VS1).

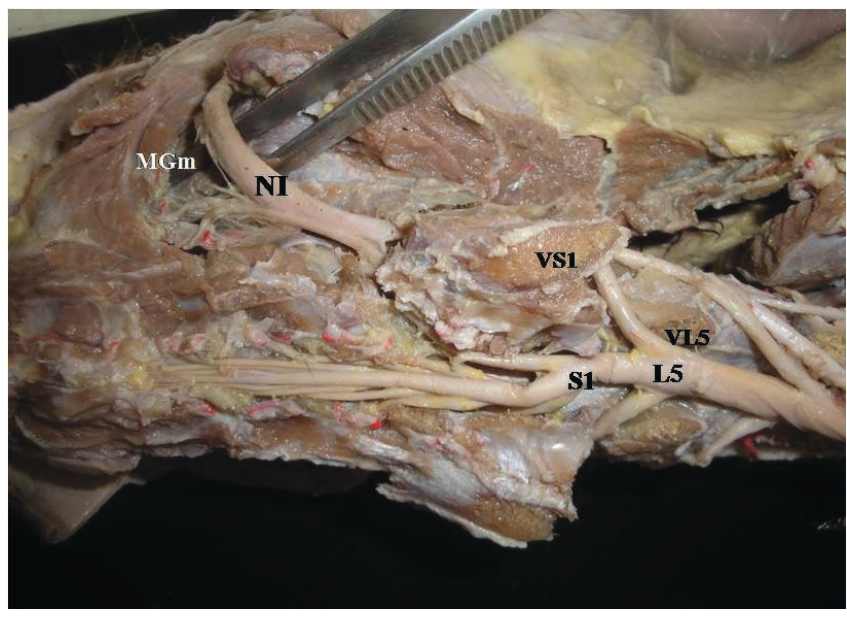

FIGURA 4: Fotomacrografia do plexo lombossacral do Leopardus pardalis (vista lateral), em que se observa a origem do nervo isquiático $(\mathrm{NI})$, e seus ramos nervo tibial (NT) e fibular comum (FC), cruzando por cima da cabeça lateral do músculo gastrocnêmio $(\mathrm{MGcl})$.

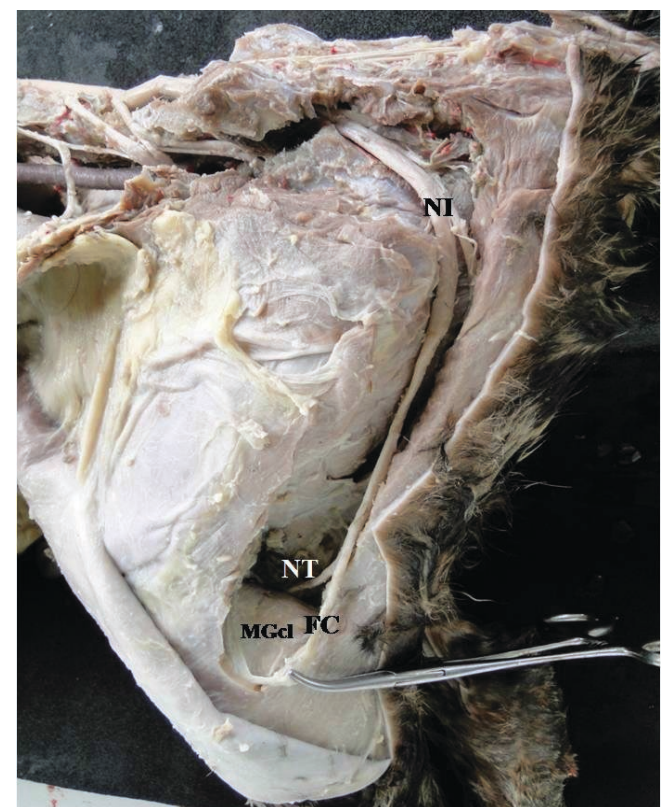

FIGURA 5: Fotomacrografia do plexo lombossacral do Leopardus pardalis (vista lateral), mostrando os nervos glúteo cranial (GCr) e nervo glúteo caudal (GCd) se originando do nervo isquiático $(\mathrm{NI})$, inervando o músculo glúteo médio (MGm) e músculo glúteo profundo (MGp).

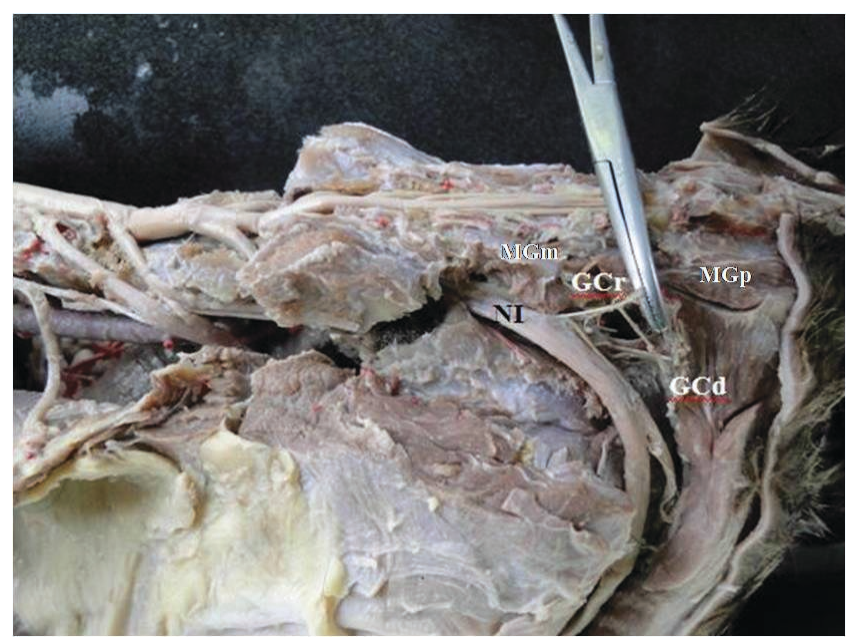

\section{Discussão}

O estudo do plexo lombossacral é de extrema importância na medicina em geral, pois é de interesse para a clínica cirúrgica, existindo estudos anestésicos em humanos, mostrando que o bloqueio do plexo lombar é 
uma técnica útil em combinação com a anestesia geral quando a anestesia peridural estiver contra-indiciada (DUARTE et al., 2009).

A origem espinhal do plexo lombossacral, o qual subsidia o membro pélvico tem sido alvo de vários estudos envolvendo diversos mamíferos como ratos, camundongos, cobaias, coelhos, mocó, paca, porcoespinho, lobo marinho, esquilo-vermelho, cães e gatos (MILLER et al., 1964; COOK, 1965; GHOSHAL, 1972; BARONE et al., 1973; COOPER; SCHILLER, 1975; GETTY, 1975; CROUCH, 1985; SCHMALBRUCH, 1986; MCLAUGHLIN; CHIASSON, 1987; NUNES; SILVINO, 1996; ASATO et al., 2000; GREENAWAY et al., 2001; LACERDA et al., 2006; AYDIN, 2009; 2010; AYDIN et al., 2009; CASTRO et al., 2009; MARTINEZPEREIRA; RICKES, 2011), porém, não há registros envolvendo felinos selvagens.

De acordo com a literatura envolvendo animais domésticos em geral, são considerados participantes do plexo lombossacral os nervos: femoral, obturador, isquiático e fibular (HABEL; ROBERTY, 1986), porém, Frandson (1979) descreveu os nervos: glúteo cranial, glúteo caudal, femoral, obturador, isquiático e fibular, como integrantes deste plexo nos animais de fazenda.

No gato doméstico (Felis catus domesticus), o plexo lombossacral é formado pelos ramos ventrais do sexto e sétimo nervos lombares e pelo primeiro nervo sacral (L6-S1). O segundo nervo sacral pode contribuir com um pequeno ramo para a sua formação. O nervo glúteo cranial deriva suas fibras dos ramos ventrais do sexto e sétimo nervos lombares. O nervo isquiático, o qual é o maior nervo do corpo dos felinos domésticos, sofrendo injúrias frequentes (CROUCH, 1969), recebe fibras dos ramos ventrais do sexto e sétimo nervos lombares e do primeiro nervo sacral. Ele deixa a cavidade pélvica por meio do forame isquiático maior e situa-se entre o músculo piriforme e o músculo glúteo profundo (CIDRAL et al., 2010). Já no Leopardus pardalis foram encontradas diferenças, de forma que o plexo lombossacral era formado por uma intercomunicação dos dois últimos nervos lombares e dos primeiros sacrais (L4-S2) e o nervo glúteo cranial foi encontrado originando do nervo isquiático, que se originou do quinto nervo lombar e primeiro e segundo nervo sacral. Tal ocorrência se deu de forma diferenciada no porco-espinho, o qual teve o nervo glúteo cranial originado de L3 e L4 (AYDIN, 2009).

Em cães, o plexo lombossacral é uma intercomunicação dos cinco últimos nervos lombares e três primeiros sacrais (L3-S3) (MILLER et al., 1964). Na jaguatirica (Leopardus pardalis), encontrou-se uma intercomunicação dos dois últimos nervos lombares e dos primeiros sacrais (L4-S2), como encontrado por Dyce (2004), qual afirmou também que o nervo femoral apresentou origem de três nervos (L4-L6). Já Schwarze e Schröder (1970) e Rocha (2003) citam que o nervo femoral nos cães é formado por dois segmentos (L4-L5), diferente do Leopardus pardalis no qual o nervo citado originou-se do quarto nervo lombar (L4).

$\mathrm{Na}$ L. pardalis, os nervos femoral, nervo obturador, isquiático, glúteo cranial e glúteo caudal, originan-se de L4, L5, L5-S1 e nervo isquiático respectivamente, fato que é diferenciado dos achados em mocó, no qual o nervo femoral parte de L4-L7, nervo obturador parte de L5-L7, nervo isquiático de L5-S2, nervo glúteo cranial de L6-L7 e nervo glúteo caudal de L6-S1 (LACERDA et al., 2006), porém, assemelha-se aos achados de Castro et al. (2007) em lobos-marinhos.

Os nervos femoral, obturador e isquiático da jaguatirica, originam-se da mesma região que foi descrita em alguns roedores como o esquilo-vermelho e a chinchila, ou seja, nervo femoral e obturador originamse juntos a partir de L4-L5, e isquiático a partir de L5S1 (AYDIN, 2010; MARTINEZ-PEREIRA; RICKES, 2011). Este último assemelhou-se com os achados de Oliveira et al. (2010), o qual descreveu que no preá o nervo isquiático originou-se de raízes ventrais de L6, L7 e S1, mas também de L7, S1 e S2.

O plexo lombossacral da L. pardalis possui diferenças em disposição e inserção com os carnívoros domésticos (cão e gato) e outros mamíferos selvagens, como descritos neste trabalho. Assim, enriquecemos o estudo morfológico para procedimentos cirúrgicos mais seguros e contribuímos para que futuros estudos sejam desenvolvidos complementando a literatura para felinos selvagens.

\section{Referências}

ASATO F.; BUTLER M.; BLOMBERG H.; GORDH T. Variation in rat sciatic nerve anatomy: implications for a rat model of neuropathic 
pain. Journal of the Peripheral Nervous System, Rochester, v. 5, p. 19-21, 2000.

AYDIN, A. The dissemination of the pelvic limb nerves originating from the lumbo-sacral plexus in the porcupine (Hystrix cristata). Veterinárni Medicine, Prague, v. 54, p. 333-339, 2009.

AYDIN, A. The spinal nerves that constitute the plexus lumbosacrales of the red squirrel (Sciurus vulgaris). Veterinárni Medicine, Prague, v. 55, p. 183-186, 2010.

AYDIN, A.; DINC, G.; YLMAZ, S. The spinal nerves that constitute the plexus lumbo-sacrales of porcupines (Hystrix cristata). Veterinárni Medicine, Prague, v. 54, p. 194-197, 2009.

BARONE, R.; PAVAUX, C.; BLIN, P. C.; CUQ, P. Atlas of rabbit anatomy. Paris: Masson et Cie, 1973. $220 \mathrm{p}$.

CASTRO, T. F.; PEREIRA, P. C. G.; PEREIRA, M. A. M.; BOMBONATO, P. P.; RICKES, E. M. Formation of the portal venous system in Chinchilla lanigera. Brazilian Journal of Veterinary Research and Animal Science, São Paulo, v. 44, p. 44-48, 2007.

CASTRO, T. F.; SOUZA, D. A. S.; SILVA FILHO, R. P.; PEREIRA, M. A. M. Sistematização e distribuição da inervação lombar e sacral em Arctocephalus australi. Brazilian Journal of Veterinary Research and Animal Science, São Paulo, v. 46, n. 5, p. 404-411, 2009.

CIDRAL, A. H.; VANDERLEI, S. R. S.; AMORIM, M. J. A. A. L.; SILVA, L. T. R.; COSTA, E. F.; SANTOS, C. R. O.; ROLIM, V. P.; OLIVEIRA, J. M. R. P. B.; BARRETO, M. L. M.; ROCHA, N. L. F. C. Estudo neuroanatômico do plexo lombossacral do gato desenvolvido pelos monitores nas aulas de Anatomia Descritiva dos Animais Domésticos. JORNADA DE ENSINO, PESQUISA E EXTENSÃO - JEPEX 2010 - UFRPE, X, 2010, Recife. Anais... Recife: UFRPE, 2010. Versão eletrônica.

COOK, M. J. The anatomy of the laboratory mouse. New York: Academic Press, 1965. 143 p.

COOPER, G.; SCHILlER, A. L. Anatomy of the guinea pig. Cambridge: Harvard University Press, 1975. 392 p.

CUBAS, Z. S. Tratado de animais selvagens. São Paulo: Roca, 2007. 1354 p.

CROUCH, J. E. Text-atlas of cat anatomy. Philadelphia: Lea \& Febiger, 1969. 399 p.

DUARTE, L. T. D.; BERALDO, P. S. S.; SARAIVA, R. A. Anestesia peridural lombar ou bloqueio do plexo lombar combinados à anestesia geral: eficácia e efeitos hemodinâmicos na artroplastia total do quadril. Revista Brasileira de Anestesiologia, Rio de Janeiro, 2009; v. 59; n. 6; p. 649-664.

DYCE, K. M.; SACK, W. O.; WENSING, C. J. G. Tratado de anatomia veterinária. 3. ed. Rio de Janeiro: Elsevier, 2004. 813 p.

FRANDSON, R. D. Anatomia e fisiologia dos animais domésticos. 2. ed. Rio de Janeiro: Guanabara Koogan, 1979. 61 p.

GETTY, R. Anatomia dos animais domésticos. 5 ed. Rio de Janeiro: Guanabara Koogan, 1975. 2048 p.

GHOSHAL, N. G. The lumbosacral plexus (plexus lumbosacrales) of the cat (Felis domestica). Anatomischer Anzeiger, Amsterdam, v. 131, p. 272-279, 1972.

GREENAWAY, J. B.; PARTLOW, G. D.; GONSHOLT, N. L.; FISHER, K. R. Anatomy of the lumbo-sacral spinal cord in rabbits. Journal of the American Animal Hospital Association, Missouri. v. 37, p. 27-34, 2001.
HABEL, A. L.; ROBERTY, W. B. Appliedy veterinaria anatomy. Washington: Saunders Company, 1986. 330 p.

INTERNATIONAL COMMITTEE ON VETERINARY GROSS ANATOMICAL NOMENCLATURE. Nomina Anatômica Veterinária. 5. ed. Knoxville: World Association on Veterinary Anatomist, 2005. 190 p.

LACERDA, P. M. O.; MOURA, C. E. B.; MIGLINO, M. A.; OLIVEIRA, M. F.; ALBUQUERQUE, J. F. G. Origin of lumbar sacral plexus of rock cavy (Kerodon rupestris). Brazilian Journal of Veterinary Research and Animal Science, São Paulo. v. 43, p. 620-628, 2006.

MCLAUGHLIN, C. A.; CHIASSON, R. B. Laboratory anatomy of the rabbit. Dubuque: W. C. Brown Company, 1987. 124 p.

MACHADO, A. B. M.; FONSECA, G. A. B.; MACHADO, R. B.; AGUIAR, L. M. S.; LINS, L. V. (Ed.). Livro vermelho das espécies ameaçadas de extinção da fauna de Minas Gerais. Belo Horizonte, Fundação Biodiversitas, 1998. 605 p.

MARTINEZ-PEREIRA, M. A.; RICKES, E. M. The spinal nerves that constitute the lumbosacral plexus and their distribution in the chinchilla. South African Veterinarian Association, Johannesburg, v. 82, n. 3, p. 150-154, 2011.

MILLER, M.; CHRISTENSEN, G.; EVANS, H. Anatomy of the dog. Philadelphia: W. B. Saunders, 1964. 941 p.

NOWELL, K.; JACKSON, P. Wild cats: status survey and conservation action plan. Gland: Internacional Union for Conservation of Nature, 1996. 382 p.

NUNES, L. C.; SILVINO, M. J. Formação do plexo lombossacral em cutia dourada (Dasyprocta aguti). In: CONGRESSO BRASILEIRO DE ANATOMIA, XVII, 1996, Fortaleza. Anais... Fortaleza: SBA, 1996. Versão eltrônica.

OLIVEIRA, G. B.; RODRIGUES, M. N.; SOUSA, E. S.; ALBUQUERQUE, J. F. G.; MOURA, C. E. B.; AMBRÓSIO, C. E.; MIGLINO, M. A.; OLIVEIRA, M. F. Origem e distribuição dos nervos isquiáticos do preá. Ciência Rural, Santa Maria, v. 40, n. 8, p. 1741-1745, 2010.

OLIVEIRA, T. G. Neotropical cats: ecology and conservation. São Paulo: EDUFMA, 1994. 220 p.

OLIVEIRA, T. G.; CASSARO, K. Guia de identificação dos felinos brasileiros. 2. ed. São Paulo: Sociedade de Zoológicos do Brasil, 1999. $60 \mathrm{p}$.

OLIVEIRA, T. G.; CASSARO, K. Guia de campo dos felinos do Brasil. São Paulo: Instituto Pró Carnívoros, Fundação Parque Zoológico de São Paulo, Sociedade de Zoológicos do Brasil, Próvida Brasil, 2005. 80 p.

REDFORD, K. H.; EISENBERG, J. F. Mammals of the Neotropics. The Southern Cone. v. 2. London: University Chicago Press, 1992. $430 \mathrm{p}$.

ROCHA, L. M. S. Estudo anátomo-anestesiológico do segmento lombar (L1 a L6) em cães. 2003. 81 f. Dissertação (Mestrado) Faculdade de Medicina Veterinária e Zootecnia, Universidade de São Paulo, São Paulo, 2003.

SCHMALBRUCH, H. Fiber composition of the rat sciatic nerve. Anatomical Record, Rochester, v. 215, p. 71-81, 1986.

SILVA, F. Mamíferos Silvestres do Rio Grande do Sul. Porto Alegre: Fundação Zoobotânica do Rio Grande do Sul, 1984.

SCHWARZE, E.; SCHRÖDER, L. Compendio de anatomia veterinaria. v. 4. Zaragoza: Acribia, 1970. 208 p. 